\title{
Treatment of postoperative sternal dehiscence with mediastinitis: Twenty-four-year use of a single method
}

\author{
J. Ernesto Molina, MD, PhD, Elaine C. Nelson, RN, CNS, and Robin R. A. Smith, RN, CNP
}

Objective: Postoperative deep sternal wound infection with dehiscence carries a high mortality rate, a high morbidity rate, and a poor cure rate. We developed a standard protocol of care to treat this complication, achieving primary closure and cure of the infection.

Methods: From January 1, 1981, through May 31, 2005 (24 years 5 months), we treated 114 patients with dehiscence and mediastinitis. The diagnosis was made from 4 to 56 days (mean, 14.5 days) after surgery. Mean age was 64.3 years (range, $38-84$ years); 79 (69\%) were obese, and 48 (42\%) had diabetes. Ten had previous attempts (1-4) of repair with other methods. Treatment entailed (1) debridement without removal of bone, (2) bilateral dissection of skin and subcutaneous tissue as one layer, (3) implantation of a staggered double-tube irrigation-suction system posterior and another one anterior to the sternum, (4) lateral reinforcement of the sternum and reclosure with a double wire, and (5) a single-layer closure of the subcutaneous tissue and skin.

Results: Of 114 patients, 109 (96\%) had mediastinitis, positive for Staphylococcus species in 101 (92.6\%). The cure rate was 98\% (112/114); hospital stay was 14 days (range, 12-16 days), with no deaths.

Conclusions: Use of this standard protocol is effective and highly recommended. It spares the sternum, cures the infection, and leaves the patient physically functional without the use of soft tissue flaps.

From the Department of Surgery, Division of Cardiothoracic Surgery, University of Minnesota Medical School, Minneapolis, Minn.

Received for publication March 10, 2006; revisions received May 5, 2006; accepted for publication June 7, 2006.

Address for reprints: J. Ernesto Molina, $\mathrm{MD}, \mathrm{PhD}$, Division of Cardiothoracic Surgery, 420 Delaware St. SE/MMC 107, Minneapolis, MN 55455 (E-mail: molin001@umn.edu).

J Thorac Cardiovasc Surg 2006;132:782-7

$0022-5223 / \$ 32.00$

Copyright $(\odot) 2006$ by The American Association for Thoracic Surgery

doi:10.1016/j.jtcvs.2006.06.008
$\mathrm{P}$ ostoperative sternal dehiscence with mediastinitis is a serious complication that needs to be treated promptly and properly. ${ }^{1-5}$ If effective treatment is not implemented in a timely fashion and for an adequate period, further complications (such as sepsis, respiratory insufficiency, or multiorgan failure) will develop and the patient may die. ${ }^{5-9}$ Such dire consequences are possible even when drastic operations are undertaken that include removal of the sternum or use of muscular or soft tissue flaps to achieve healing. The likelihood of patient survival is limited. ${ }^{6}$

Keeping these concepts in mind, we designed a standard protocol of treatment implemented continually since $1981 .^{3-10}$ This report entails a consecutive unselected series of patients either operated on by the first author (J.E.M.) or referred to us by the other surgeons of our staff or by surgeons outside our institution. All comers were treated with the same protocol with no exceptions regardless of the age of the dehiscence. Our protocol aims to cure the infection and to re-establish normal chest stability without the use of muscle or omental flaps, sparing the sternum and leaving the patient anatomically intact and fit for normal activities.

Although other irrigation/suction techniques have been used in the past, ${ }^{11-14}$ their failure rates remained high. ${ }^{5,12}$ We herein present a complete review of our results since our original publication, back in $1993 .{ }^{10}$ 
TABLE 1. Operations performed on patients treated with the protocol

\begin{tabular}{lr}
\hline Operation & No. \\
\hline CAB & 91 \\
AVR & 6 \\
AVR + CAB & 3 \\
Asc.Ao.Aneu. & 2 \\
CAB + MVR & 2 \\
MVR & 4 \\
Heart transplant & 2 \\
AVR + MVR & 2 \\
TOF & 1 \\
AV canal & 1 \\
Total & 114
\end{tabular}

$\overline{C A B}$, Coronary artery bypass; $A V R$, aortic valve replacement; $A s c$. Ao.Aneu, ascending aortic aneurysm; $M V R$, mitral valve replacement; $T O F$, tetralogy of Fallot; AV canal, atrioventricular canal.

\section{Patients and Methods}

From January 1, 1981, through May 31, 2005 (24 years 5 months), we treated a consecutive unselected series of 114 patients with postoperative sternal dehiscence that occurred from 4 to 56 days after surgery (mean, 14.5 days). Several patients had already been discharged after their initial hospitalization and were readmitted when their sternal incision became unstable or started draining or both. Three patients were septic with bacteremias, 2 with Staphylococcus infection and 1 with Serratia. Mean age was 64.3 years (range, 38-84 years). Obesity (body mass index $>30$ ) was present in $79(69 \%), 48(42 \%)$ had diabetes, 4 (3.5\%) severe chronic obstructive pulmonary disease, $3(2.6 \%)$ had mild renal failure, and $1(0.8 \%)$ was receiving long-term steroid therapy. The diagnosis of mediastinitis was made by the physical presence of purulent material in the field, either preoperatively when the incision was already open and draining, where separation of the sternal edges allowed visualization of the mediastinum, or at the time of surgery when purulent material was found behind the sternum or cultures from the mediastinum returned positive. The types of operations performed in these patients are shown in Table 1 .

\section{Operative Technique}

Our protocol consists of the following steps:

After the incision is reopened to its original length, all wires are removed. The entire field is irrigated with a high-pressure system (Simpulse pulsed lavage; Davol Inc, Cranston, RI) or similar, containing antibiotic solution (usually $1 \mathrm{~g}$ of cephalosporin in 1000 $\mathrm{mL}$ of saline solution). The skin and subcutaneous tissue are undermined as a single layer on both sides until the lateral border of the sternum is reached (Figure 1). The pectoralis major muscle is not mobilized.

The adhesions behind the sternum are taken down until the lateral border of the sternum is passed bilaterally. Each intercostal space is clearly identified. All granulation tissue is removed with a curette instrument. High-pressure irrigation is applied to the bone and the retrosternal space.

A reinforcing single strand wire with a blunt needle, gauge 8 (A\&E Medical Corp, Durham, NC), is laced lateral to the sternum

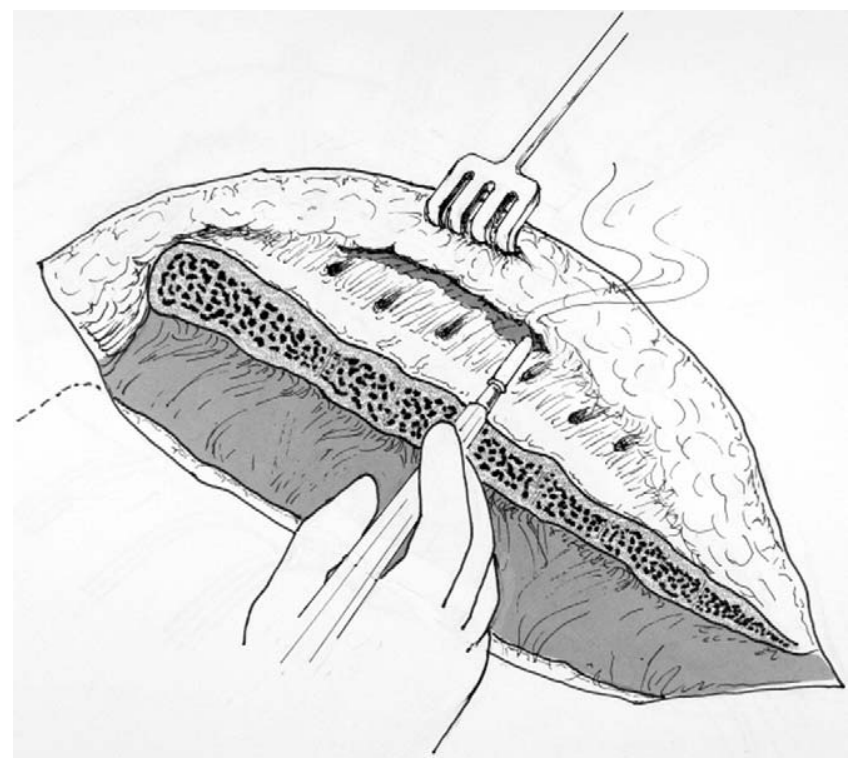

Figure 1. The first stage of the repair after all the wires have been removed. The skin and subcutaneous tissue are dissected laterally as a single layer until the lateral border of the sternum is past and the intercostal spaces are properly identified.

(Figure 2), around each rib. The wire starts at the costal margin, extends up to the manubrium, and returns in reverse order, as described by Robicsek, Dougherty, and Cook, ${ }^{15}$ so that each rib is surrounded by wire anteriorly and posteriorly. In addition, a hole is drilled in the upper portion of the manubrium to allow placement of the reinforcing wire up to that level. At the lower end (ie, the costal margin), both ends are twisted and buried in the deep tissues. No bone is removed.

A posterior system of staggered chest tubes is placed along the length of the sternum. Two size- 24 chest tubes for suction and 2 small (gauge 8 or 10) catheters for irrigation are placed parallel to each chest tube (Figure 3). Peristernal encircling double wires, gauge 8 (A\&E Medical Corp), are placed lateral to the reinforcing wires (Figure 3 ) and tightened. The sternum is reunited. Superficial to the sternum, 2 size- 20 chest tubes are positioned in a staggered manner also, accompanied by 2 irrigating catheters (gauge 8 or 10) along the entire length of the sternum (Figure 4). The skin and subcutaneous tissue are closed in a single layer with a continuous suture: heavy, nonabsorbable thick monofilament material (Number 2 Prolene; Ethicon, Inc, Somerville,NJ) is used (Figure 4).

\section{Postoperative Care}

The patients are extubated after surgery or the next morning. Normal oral feedings are resumed. Continuous irrigation at 100 $\mathrm{mL}$ per tube per hour is applied around the clock for 7 days. Independent infusion pumps (4 total) should be used to prevent uneven irrigation. Y-connectors are not used. Thus, the total should be $400 \mathrm{~mL} / \mathrm{h}$ for all 4 irrigating catheters. The only disadvantage of this large-volume system is that the containers collecting the irrigation need to be changed often. Currently, all patients are given low-molecular-weight heparin (Lovenox, Sanofi Aventis, 


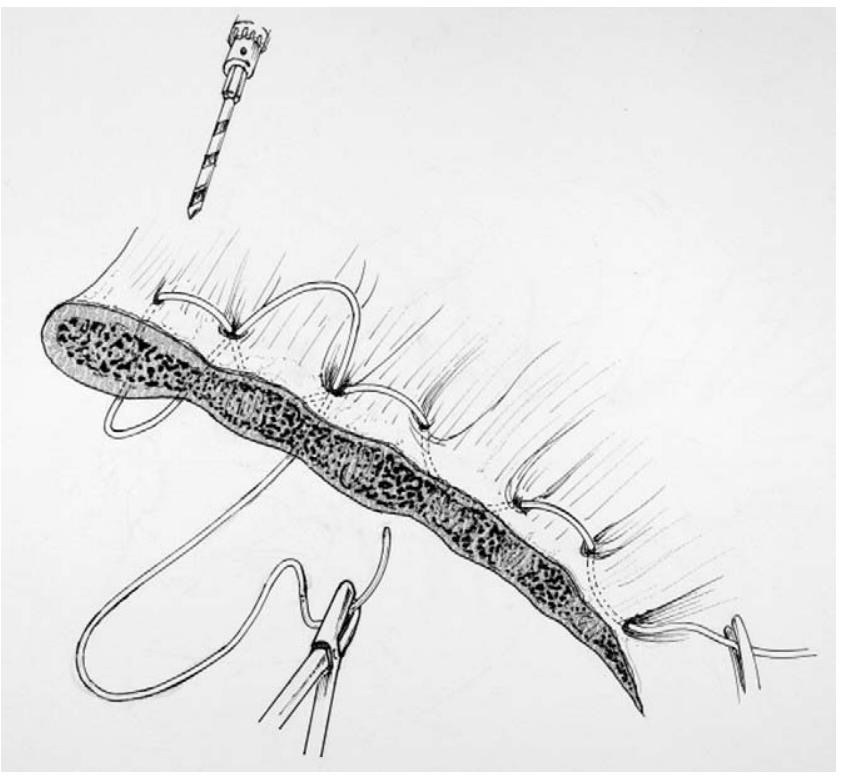

Figure 2. The reinforcing wire placed parallel to the sternum is laced around each rib from the costal margin to the manubrium and back. A hole is drilled in the upper part of the manubrium to allow the parallel reinforcing wire to reach that level to allow the placement of the peristernal wires embracing the reinforcing wire up to that level.

Bridgewater, NJ; enoxaparin), $40 \mathrm{mg}$ twice a day, and wear pneumoboots as long as they are bedridden to prevent deep vein thrombosis and pulmonary embolism. In our earlier experience, unfractioned heparin (5000 IU bid subcutaneously) was used, but since 1994 enoxaparin has been adopted.

Patients are kept on complete bed rest, with the bed flat; head elevation is allowed only to $12^{\circ}$, with no sitting and no Fowler position permitted (to avoid kinking of the tubes or increased tension of the skin edges). The bladder catheter remains in place for the first week. If, by the end of 48 hours, the fluid return is clear with no fibrinous material, clots, or debris, the irrigation rate can be decreased to either 75 or $50 \mathrm{~mL}$ per tube per hour until the end of the week. At 1 week, irrigation is discontinued, and all irrigating catheters are removed. At this point, patients are allowed to get out of bed. To protect the stability of the repair, patients are allowed only to extend their arms in front of them; if they want to get off the bed, they must be gently pulled, by an assistant standing at the foot of the bed, into a sitting position, and then swing themselves around. Patients are not allowed to push with their arms sideways or from the back.

During the following week (ie, postoperative week 2) the suction tubes are advanced out 2 inches every day until they are all out (usually over 5 to 7 days).

Patients continue to receive the proper intravenous antibiotic therapy. Because most of the infections seen in our series were due to Staphylococcus species, our antibiotic of choice is vancomycin. For the topical irrigation antibiotic solution, cefazolin at $1 \mathrm{~g} / 1000$ $\mathrm{mL}$ of solution is used. However, this is changed to vancomycin or

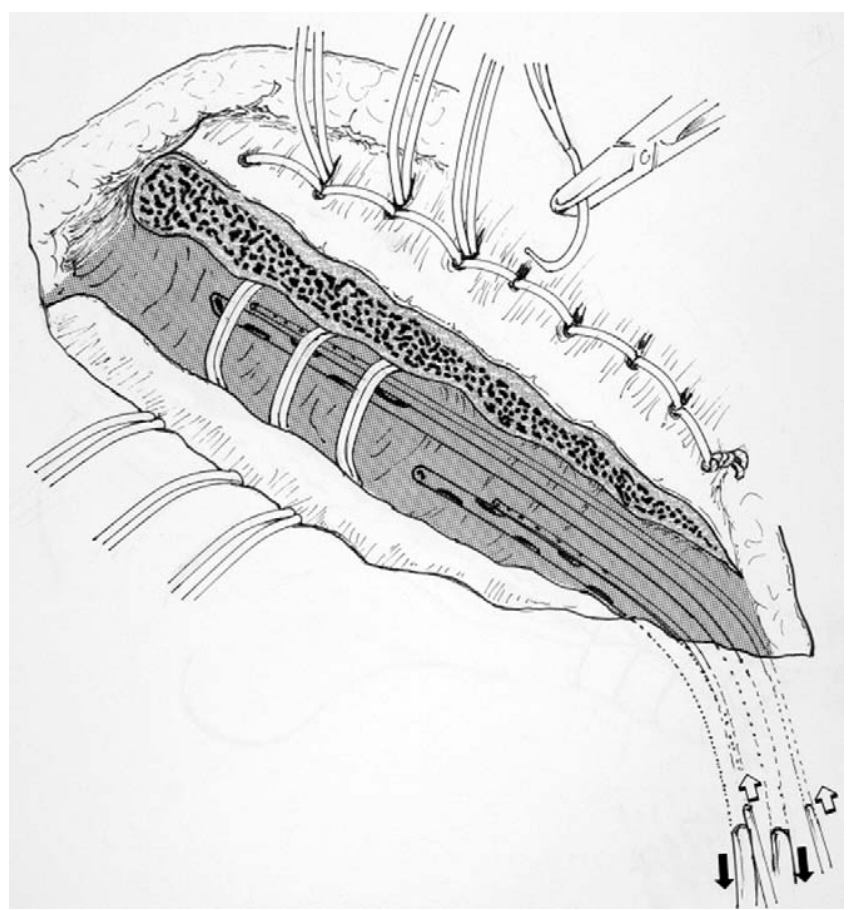

Figure 3. The retrosternal chest tubes are placed accompanied by an irrigation catheter in a staggered manner reaching up to the sternal notch. The double peristernal wires are now passed lateral to the reinforcing laced wire to close the sternum.

gentamicin if the organism is resistant to cephalosporins. The patients who had gram-negative or mixed infections received intravenous and irrigation of aztreonam (Azactam, Bristol-Myers Squibb, Princeton, NJ), piperacillin and tazobactam (Zosyn, Wyeth Pharmaceuticals, Philadelphia, Pa), gentamicin, or ampicillin and sulbactam (Unasyn, Pfizer Inc, New York, NY). Whichever antibiotic was chosen, the treatment was implemented for the same length of time: 2 weeks while in the hospital, then orally or intravenously via a peripherally inserted central catheter for the third week (after hospital discharge) only when vancomycin was required.

Twice a day, the skin incision is cleansed and rinsed with either Techni-Care surgical scrub (Care-Tech Laboratories, Inc, Saint Louis, Mo) or chlorhexidine topical (Hibiclens, Regent Medical, Cheshire, UK) to prevent build-up of debris or dried-up crust material that may harbor bacteria. No dressings are applied. After all tubes are out, patients are discharged home and continue with antibiotic therapy, this time orally for another 10 days. The monofilament sutures closing the skin should stay for a total of 3 weeks. The long-term follow-up requires only 2 visits to the clinic: at 1 week after discharge to remove the skin stitches, then 6 weeks after that for the final check-up. Cure results were assured at 2 months' follow-up.

\section{Results}

Of all 114 patients, 109 (96\%) had mediastinitis as previously defined. The other 5 patients had open skin incision, 


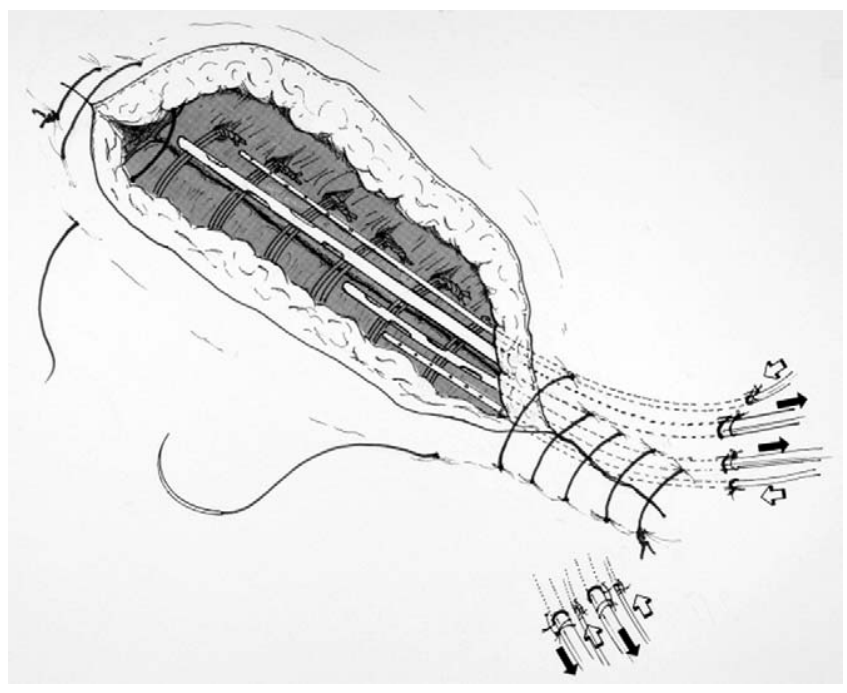

Figure 4. After the sternum is closed, a superficial system of chest tubes, also placed in a staggered manner with irrigating catheters, is laid over the sternum and brought out on the left side of the upper abdomen. The skin and subcutaneous tissue are closed on one single layer with monofilament suture (Number 2 Prolene suture).

separation of the sternum, and loose wires with fractures but no obvious pus. The most common cultured organisms were Staphylococcus species in 101 patients (92.2\%): $78 \mathrm{~S} \mathrm{epi-}$ dermidis and $23 \mathrm{~S}$ aureus, of which 4 were methicillin resistant (Table 2). The remaining 8 patients had either a mixed flora (4 patients.), gram-negative organisms (2 patients.), Enterococcus, or no detectable growth in 1 patient, probably because of a prolonged course of antibiotics to which he had been subjected. Only 1 death occurred in the entire series, caused by heart failure 2 months after the sternal dehiscence repair.

Of the 114 patients, $102(90 \%)$ had at least 1 fracture. We found from 1 to 6 fractures per patient counting both sides of the sternum at the time of the exploration (Table 3 ). In the

TABLE 2. Infective organisms in the patients with postoperative sternal dehiscence with mediastinitis

\begin{tabular}{lrc}
\hline Organism & No. & Percent \\
\hline Staphylococcus species & 101 & $92^{*}$ \\
Mixed florat & 4 & 3.6 \\
Serratia & 1 & 0.9 \\
Enterococcus & 1 & 0.9 \\
Enterobacter & 1 & 0.9 \\
No growth in culture & 1 & 0.9 \\
Total & 109 &
\end{tabular}

*Includes 2 patients with baceremia. †Klebsiella, Mycoplasma, Corynebacterium, Pseudomonas, and Staphylococcus species. presence of a fracture, we carried out the reinforcement by placing the wire parallel to the sternum just as if the sternum were intact. We made sure that the suture line was maintained snug in both directions to keep the fragments of sternum approximated as well as possible.

The cure rate was $98 \%(112 / 114$ patients) with a completely healed stable sternum and no recurrences. In the other 2 patients our repair failed after two previous rewiring repairs had been attempted with different methods. Eventually they underwent removal of the sternum and muscle flap implants. The mean hospital stay was 14 days (range, 12-16 days). No deaths and no disabilities occurred as a result of the repair. However, 3 patients treated with our protocol needed another reintervention because the first time the repair had been inadequate and loose, with wires improperly placed around the sternum. All 3 patients underwent reoperation with the same protocol and all of them recovered satisfactorily.

Two patients who were referred late (49 to 56 days postoperatively) had been diagnosed elsewhere with osteomyelitis and recommended to have the sternum removed. Both had chronic multiple open draining sites. We conducted our standard method and obtained total cure. One other patient (48 days postoperatively) had also undergone a previous repair attempt with a pectoralis major muscle flap and rewiring that failed. This patient was also cured.

There were 10 patients $(8.7 \%)$ with sternal dehiscence who had undergone previous (1 to 4 ) attempts at repairs using other rewiring techniques that failed before they were referred to have our protocol of care implemented. Our technique was successful in 8 with total recovery. The 2 failures were the only ones that occurred in our series.

In this series no deep vein thrombosis or pulmonary embolism occurred in any patient. Therefore, the use of enoxaparin (Lovenox) seems to be very effective to prevent these complications.

No pulmonary complications were seen. On the contrary, some of the patients who were experiencing respiratory embarrassment preoperatively while the sternum was unstable had significant improvement once the sternum was stabilized. No prolonged respirator dependency was observed after the sternal repair. The patients who needed anticoagulation therapy for mechanical valve prosthesis im-

TABLE 3. Distribution of sternal fractures in $102(89.4 \%)$ of 114

\begin{tabular}{lc}
\hline No. of fractures & Total on either side or both \\
\hline 1 & 34 \\
2 & 42 \\
3 & 19 \\
4 & 7 \\
Total & 102 \\
\hline
\end{tabular}



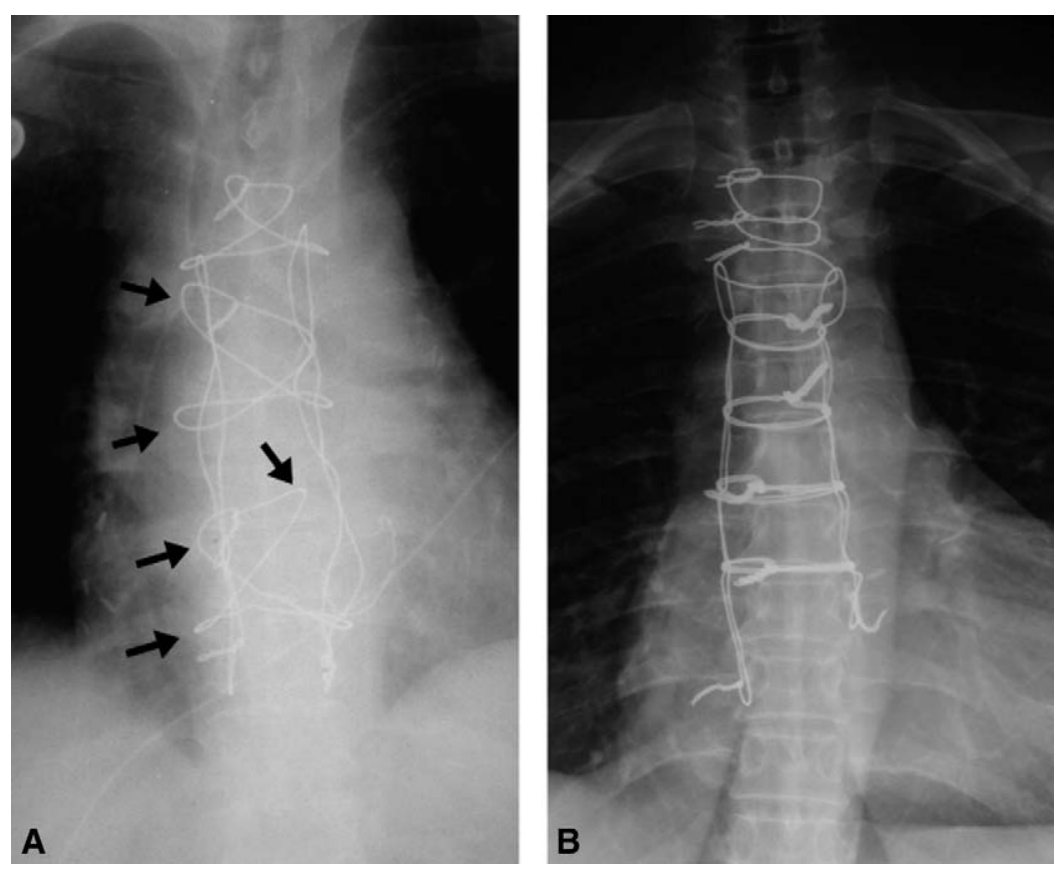

Figure 5. A, The reinforcing and reclosure of the sternum has been done inappropriately. The peristernal wires are loose in several places (arrows), making them ineffective in bringing the sternum together using the support of the parallel reinforcing wire suture. B, Correct reinforcement of the sternum is shown, with the peristernal wires properly tightened against the parallel reinforcing wires.

plants or atrial fibrillation were restarted on warfarin (Coumadin, Bristol-Myers Squibb) the day after surgery. No bleeding episode or systemic embolism occurred in these patients. No case of decubitus ulcer developed since the period of bed rest is relatively short (7 days). The patients are also rotated in bed from side to side.

\section{Discussion}

Postoperative sternal dehiscence continues to occur when midline sternotomy is used to approach the mediastinal structures. Its occurrence is frequently associated with infection, namely, mediastinitis, which is a serious complication with high mortality. The protocol of care presented here was designed not only to cure the infection, but also to restore the integrity of the sternum. It is a tedious operation and difficult, particularly the retrosternal dissection to accomplish the repair. Even though the sternum is firm and stable after this repair, bed rest is necessary to implement our method of treatment because the patient cannot be mobilized with the multiple irrigating tubes, pumps, and suction tubes working continuously. Once the irrigation is discontinued at the end of the first week, the patient gets out of bed only with the chest tubes on suction.

Several key points in implementing this protocol require emphasis: Our most important recommendation is to re-explore the surgical field as soon as there is any suspicion that the sternum has dehisced, particularly if fever is present and/or white cell count elevation exists. Dehiscence occurs most commonly in obese patients, yet it is difficult to make a diagnosis by physical examination, because the layer of subcutaneous tissue is too thick to adequately palpate or feel movement in the sternum. Therefore, the diagnosis in obese patients is made by clinical assessment. Sometimes a computed tomographic scan of the chest may help ${ }^{16}$ by showing separation of the sternal halves. However, fluid collection as pockets is rarely seen. The longer it takes to decide for reexploration, the more fractures of the sternum will develop, thus making the repair more difficult. Another disadvantage associated with waiting is the development of severe mediastinal fibrosis. Therefore, at the time of the reintervention, taking down retrosternal adhesions and isolating the sternum is very difficult, tedious, and risky. The average time for one of these repairs is 3 to 4 hours.

Our protocol, if strictly followed, leads to total recovery. It is not advisable to "cut corners" and try to get away with a less complicated procedure, because it will most likely fail. For example, the surgeon may decide that a fewer number of tubes is satisfactory, or that a shorter treatment period is sufficient, or that all tubes can come out immediately after the first week. Also, the correct placement of the new wires is of utmost importance. Figure 5, $A$, shows improper placement of the peristernal wires after the dehiscence is treated. Figure $5, B$, shows exactly the way the $\mathrm{x}$-ray film should look after a correct repair.

We are well aware of the effectiveness of using soft tissue flaps, namely, muscles of the abdomen, chest, or even omentum. ${ }^{2,13,17}$ These procedures may cure the infections, but often they involve removal of the sternum and frequently more than one operation.

A recent modality is the use of vacuum devices on the open incision. Although beneficial, the vacuum system is only a 
TABLE 4. Timing of sternal repair after the original cardiac operation

\begin{tabular}{lcc}
\hline Postoperative days & No. of patients & Percent \\
\hline Less than 7 & 21 & 18.5 \\
$8-14$ & 39 & 34.4 \\
$15-21$ & 23 & 20 \\
$22-28$ & 12 & 10.5 \\
$29-36$ & 11 & 9.6 \\
$37-42$ & 2 & 1.7 \\
$43-48$ & 2 & 1.7 \\
$49-56$ & 4 & 3.6 \\
Total & 114 & 100 \\
\hline
\end{tabular}

bridge to later closure of the incision. Used alone, it will not render a stable sternum and it always involves two or more operations. ${ }^{18-20}$

We need to mention the type of irrigation to be used on the open sternotomy and in a closed system. Iodine compounds, mainly povidone-iodine (Betadine, Purdue Fredrick Co, Norwalk, Conn), have been shown to be dangerous if they reach serosal cavities, ${ }^{15,21}$ and deaths due to iodine toxicity have been reported. ${ }^{22}$ Iodine also inhibits fibroblasts, ${ }^{23}$ and it affects the chemotaxis of white cells, particularly in open wounds. Regardless of the type of mediastinitis or dehiscence that patients have, we apply the same technique. Therefore, the classification proposed by El Oakley and Wright ${ }^{24}$ has little application because our method has been effective for all patients, including those treated late (ie, 6 or 8 weeks after their original operation) (Table 4).

Finally, osteomyelitis is a concern. It is important to mention that all the patients treated with our protocol healed, even if the bone appeared dead, nonvascularized, or infected. Two of our patients had already been diagnosed with osteomyelitis. Both responded well. All patients healed completely.

In summary, prompt and proper implementation of our protocol is effective and therefore highly recommended for patients with sternal dehiscence and mediastinitis. Meticulously and strictly applied, this method saves the sternum and leaves the patient physically functional.

\section{References}

1. The Parisian mediastinitis study group: risk factors for deep sternal wound infections after sternotomy-a prospective, multicenter study. J Thorac Cardiovasc Surg. 1996;111:1200-7.

2. Loop FD, Lytle BW, Cosgrove DM, Mahfood S, McHenry MC, Goormastic M, et al. Sternal wound complications after isolated coronary artery bypass grafting: early and late mortality, morbidity and cost care. Ann Thorac Surg. 1990;49:179-87.

3. Molina JE, Lew RS, Hyland KJ. Postoperative sternal dehiscence in obese patients: incidence and prevention. Ann Thorac Surg. 2004;78: 912-7.
4. Prabhakar G, Haan CK, Peterson ED, Coombs LP, Cruzzavala JL, Murray GF. The risks of moderate and extreme obesity for coronary artery bypass grafting outcomes: a study from the Society of Thoracic Surgeons Database. Ann Thorac Surg. 2002;74:1125-31.

5. Trouillet JL, Vuagnat A, Combes A, Bors V, Chastre J, Gandjbakhch I, et al. Acute poststernotomy mediastinitis managed with debridement and closed-drainage aspiration: factors associated with death in the intensive care unit. J Thorac Cardiovasc Surg. 2005;129:518-24.

6. Braxton JH, Marrin CAS, McGrath PD, Morton JR, Norotsky M, Charlesworth DC, et al. 10-year follow up of patients with and without mediastinitis. Semin Thorac Cardiovasc Surg. 2004;16:70-6.

7. Levi N, Olsen PS. Primary closure of deep sternal wound infection following open heart surgery: a safe operation? J Cardiovasc Surg. 2000;41:241-5.

8. Demmy TL, Park SB, Lieber GA, Burkholder JA, Maher TD, Benckart $\mathrm{DH}$, et al. Recent experience with major sternal wound complications. Ann Thorac Surg. 1990;49:458-62.

9. Tang GHL, Maganti M, Weisel RD, Borger MA. Prevention and management of deep sternal wound infection. Semin Thorac Cardiovasc Surg. 2004;16:62-9.

10. Molina E. Primary closure for infected dehiscence of the sternum. Ann Thorac Surg. 1993;55:459-63.

11. El Gmel A, Yonan NA, Hassan R, Jones MT, Campbell CS, Deiraniya AK, et al. Treatment of mediastinitis: early modified Robicsek closure and pectoral major advancement flaps. Ann Thorac Surg. 1998;65: 41-7.

12. Calvart S, Trouillet JL, Nataf P, Vuagnat A, Chastre J, Gibert C. Closed drainage using Redon catheters for local treatment of poststernotomy mediastinitis. Ann Thorac Surg. 1996;61:195-201.

13. Pairolero PC, Arnold PC. Management of recalcitrant median sternotomy wounds. J Thorac Cardiovasc Surg. 1984;88:357-64.

14. Francel TJ, Kouchoukos NT. A rational approach to wound difficulties after sternotomy: reconstructions and long-term results. Ann Thorac Surg. 2001;72:1419-29.

15. Robicsek F, Dougherty HK, Cook JW. The prevention and treatment of sternum separation following open-heart surgery. J Thorac Cardiovasc Surg. 1977;73:267-8.

16. Misawa Y, Fuse K, Hasegawa T. Infectious mediastinitis after cardiac operations: computed tomographic findings. Ann Thorac Surg. 1998; 65:622-4.

17. Lee AB, Schimert G, Shatkin S. Total excision of the sternum and thoracic pedicle transposition of the greater omentum; useful stratagems in managing severe mediastinal infections following open heart surgery. Surgery. 1976; 80:433-6.

18. Hersh RE, Jack JM, Dahman MI, Morgan RF, Drake DB. The vacuum-assisted closure device as a bridge to sternal wound closure. Ann Plast Surg. 2001;46:250-54.

19. Fuchs U, Zittermann A, Stuettgen B, Groening A, Minami K, Koerfer R. Clinical outcome of patients with deep sternal wound infection managed by vacuum-assisted closure compared to conventional therapy with open packing: a retrospective analysis. Ann Thorac Surg. 2005;79:526-31.

20. Sjogren J, Gustafsson R, Nilsson J, Malmsjo M, Ingemansson R. Clinical outcome after poststernotomy mediastinitis: vacuum-assisted closure versus conventional treatment. Ann Thorac Surg. 2005;79: 2049-55.

21. Arenholz HD, Simmons RL. Povidone-iodine in peritonitis. I: Adverse effects of local instillation in experimental E. coli peritonitits. J Surg Res. 1979;26:458-63.

22. Glick PL, Guglielono BJ, Tranbaugh RF, Turley K. Iodine toxicity in a patient treated by continuous povidone-iodine mediastinal irrigation. Ann Thorac Surg. 1985;39:478-80.

23. Lineaweaver W, Howard RJ, Soucy D, McMorris S, Freeman J, Crain C, et al. Topical antimicrobial toxicity. Arch Surg. 1985;120: 267-70.

24. El Oakley RM, Wright JE. Postoperative mediastinitis: classification and management. Ann Thorac Surg. 1996;61:1030-6. 